\title{
Magnetic nanowires (Fe, Fe-Co, Fe-Ni) - magnetic moment reorientation in respect of wires composition
} Dariusz Satula

\begin{abstract}
Magnetic nanowires of $\mathrm{Fe}, \mathrm{Fe}-\mathrm{Co}$, and $\mathrm{Fe}-\mathrm{Ni}$ alloy and layered structure were prepared by electrochemical alternating current (AC) deposition method. The morphology of the nanowires in and without the matrix was studied by energy dispersive X-ray spectroscopy (EDX), scanning electron microscopy (SEM), and X-ray diffraction (XRD), respectively. The wires either show strong dependence on the combination of elements deposition (alloy or layered) or chemical composition (Co or Ni). The magnetic properties of the nanostructures were determined on the basis of Mössbauer spectroscopy (MS).
\end{abstract}

Key words: electrochemistry • electrodeposition • Mössbauer spectroscopy • nanowires

B. Kalska-Szostko ${ }^{\bowtie}$, U. Wykowska

Institute of Chemistry,

University of Bialystok,

1 Hurtowa Str., 15-399 Białystok, Poland,

Tel.: +48 857470113

E-mail: kalska@uwb.edu.pl

D. Satuła

Faculty of Physics,

University of Bialystok,

1L K. Ciołkowskiego Str., 15-245 Białystok, Poland

Received: 18 June 2014

Accepted: 15 November 2014

\section{Introduction}

The vast development of nanotechnology and nanoscience in the last decades was possible due to availability of the novel characterization tools and studies of new fabrication route. Huge scientific interest has been dedicated to the materials with reduced dimensionality because of their unusual physical properties and variety of potential applications. Among others, magnetic nanostructures approaching Tbit $/ \mathrm{cm}^{2}$ are seen in high-density magnetic storage devices [1]. Magnetic nanostructures are also extensively studied due to their biomedical and biotechnological applications. The special and interesting kind of magnetic nanostructures are tubular and elongated ones which become highly attractive as multifunctional spices. Special alloy wires can be used in: magnetic recording media, sensors, and inductors as well as in other functional microstructures [2]. Nanostructures with a high aspect ratio and not uniform composition can be used for multiple biological sensing, biological separations, and gene delivery [3-5]. Nanowires saturation magnetization and coercive field can be modified by the combination of different elements in one superstructure [6]. Chemical surface modification of magnetic wires can be tailored for selective interaction with molecules of special interest [7]. Magnetic nanowires can be used as skeletons of hierarchical ordering of molecules into larger superstructures for a large variety of biosensors. Besides many studies that have been done so far, there are still numerous challenges related to reproducible fabrication 

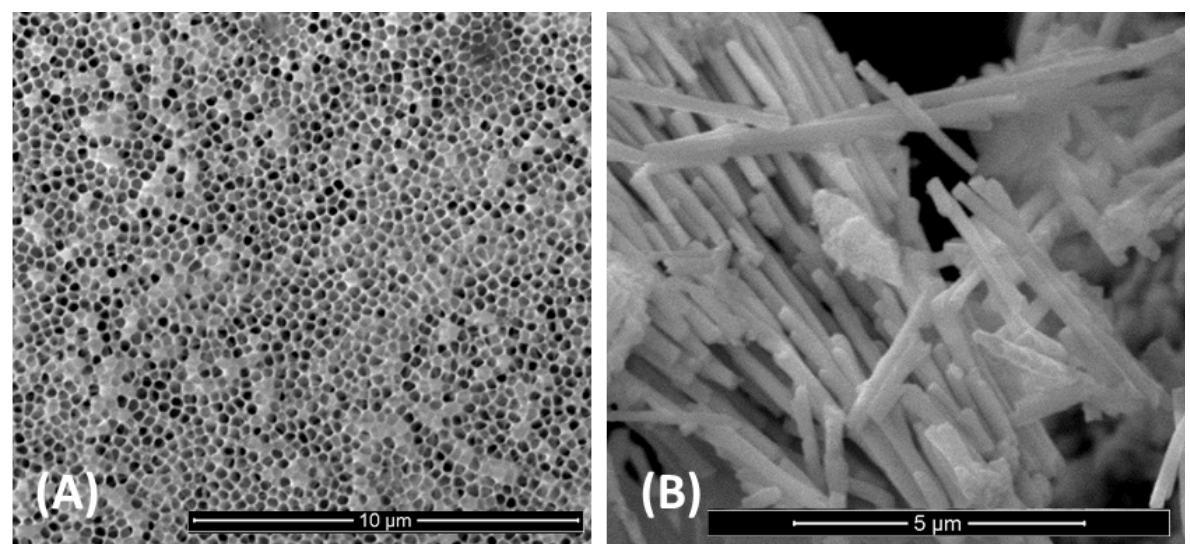

Fig. 1. SEM image of pores arrangement on alumina surface (A), and an example of deposited nanowires (B).

of nanostructures with well-defined physical and chemical properties. Therefore some difficulties still demand extensive investigations of novel materials. Wires fabrication method (electrodeposition) is very attractive in comparison to other methods because of its flexibility to the size and shape of the template [8, 9]. The element specific deposition processes were studied in case of thin films while the nanowires deposition shows much complex behavior $[6,10]$ and the mechanism of alloy nanowires deposition has not been investigated in detail so far.

In the paper we report on the electrodeposition and properties of $\mathrm{Fe}, \mathrm{Fe}-\mathrm{Ni}$, and $\mathrm{Fe}-\mathrm{Co}$ nanowires fabricated in various manners (as alloy or layered structures). Proposed wires were characterized by: $\mathrm{X}$-ray diffraction (XRD), scanning electron microscopy (SEM), energy dispersive X-ray spectroscopy (EDX), and Mössbauer spectroscopy (MS).

\section{Results and discussion}

Templates for wires deposition were obtained by anodization of aluminum in acidic solution under current conditions appropriate for the growth of the required pores diameter [11]. The details of the preparation of the matrices were described elsewhere $[12,13]$. The example of anodic alumina oxide (AAO), the template used for the fabrication of wires, is shown in Fig. 1A. Here the pores in the matrix are of the order of $(150 \pm 20) \mathrm{nm}$. The structure of the AAO template can be modified by the solution composition and voltage-current conditions. The pores diameter and their plane density are proportional to the AC voltage [14].

Magnetic nanowires have been fabricated by constant current (DC) electrochemical deposition in AAO matrix. The aluminum foil covered with AAO and platinum plate, serve as working and reference electrodes, respectively. The preparation of the wires, with various elemental compositions in layers, was realized by the sequential use of the appropriate electrolytes. For Fe-Ni wires, separate aqueous solutions of $\mathrm{FeSO}_{4} \cdot 7 \mathrm{H}_{2} \mathrm{O}$ and $\mathrm{NiSO}_{4} \cdot 7 \mathrm{H}_{2} \mathrm{O}$ were used. The average total deposition time per sample was 20 min at room temperature under applied current $10 \mathrm{~mA}$. The electrolytes for Fe-Co layered deposition contain $\mathrm{FeSO}_{4} \cdot 7 \mathrm{H}_{2} \mathrm{O}$ or $\mathrm{CoSO}_{4} \cdot 7 \mathrm{H}_{2} \mathrm{O}$ salts diluted in water. The deposition conditions were kept the same as in the case of FeNi. For all samples, the available working area was the same for the deposition $\left(1.5 \mathrm{~cm}^{2}\right)$. In case of alloy kind of wires, pairwise salts were mixed and present in the bath $\left(\mathrm{FeSO}_{4} \cdot 7 \mathrm{H}_{2} \mathrm{O}\right.$ and $\mathrm{NiSO}_{4} \cdot 7 \mathrm{H}_{2} \mathrm{O}$ or $\mathrm{FeSO}_{4} \cdot 7 \mathrm{H}_{2} \mathrm{O}$ and $\mathrm{CoSO}_{4} \cdot 7 \mathrm{H}_{2} \mathrm{O}$ ) in respective molar ratio of $1: 1$.

Prior to the characterization, the templates were kept in $1 \mathrm{M} \mathrm{NaOH}$ for alumina dissolution. The structure of the nanowires was observed by scanning electron microscope INSPCT S50. As can be seen from the presented image (Fig. 1B), the fabricated nanowires are well organized, dense, and possess smooth walls which suggest that deposition conditions were chosen in the adequate way.

To be sure that both elements are present in the wires, an example of EDX analysis was registered on SEM Microscope (TM-1000 HITACHI Tabletop) for $\mathrm{Fe}, \mathrm{Fe}-\mathrm{Co}$, and Fe-Ni system as shown in Fig. 2A. The obtained quantitative results are as follows: the fraction of Co in case of deposited alloys is slightly higher than that in the electrolytes. For Fe-Co solutions with a molar ratio of constituents in the solution 1:1, the elements ratio in the deposited wires was found to be $0.85 \pm 0.04: 1.00 \pm 0.04$, respectively. While for Fe-Ni the elements ratio was calculated to be $1.00 \pm 0.04$ : $0.95 \pm 0.04$. The results are in agreement with the observation that Co deposition is faster than deposition of $\mathrm{Fe}$ or $\mathrm{Ni}$ in case of thin films [10].

The crystalline structure of the fabricated nanowires was analyzed by X-ray diffraction. A small amount of powder was deposited on a nylon loop and analyzed in Agilent Technologies SuperNova diffractometer equipped with microfocus Mo $\left(\mathrm{K}_{\alpha}\right.$ $=0.713067 \mathrm{~nm}$ ) X-ray source. The analysis of the layered nanowires shows that the wires have been fabricated as crystalline structures with the recognized composition of used elements (see Fig. 2B). Here typical signals for bcc Fe (110) (200) (211) (220) (310) [15] can be found accompanied by additional ones which are recognized as characteristic for hexagonal Co (100) (002) (102), and fcc Ni (111) (200) (220) (311) [16-18].

Magnetic properties of the wires in the matrix were investigated by transmission Mössbauer spectroscopy. For this case a traditional set-up working in constant acceleration mode and equipped with ${ }^{57} \mathrm{Co}$ source in a $\mathrm{Cr}$ matrix was used. 


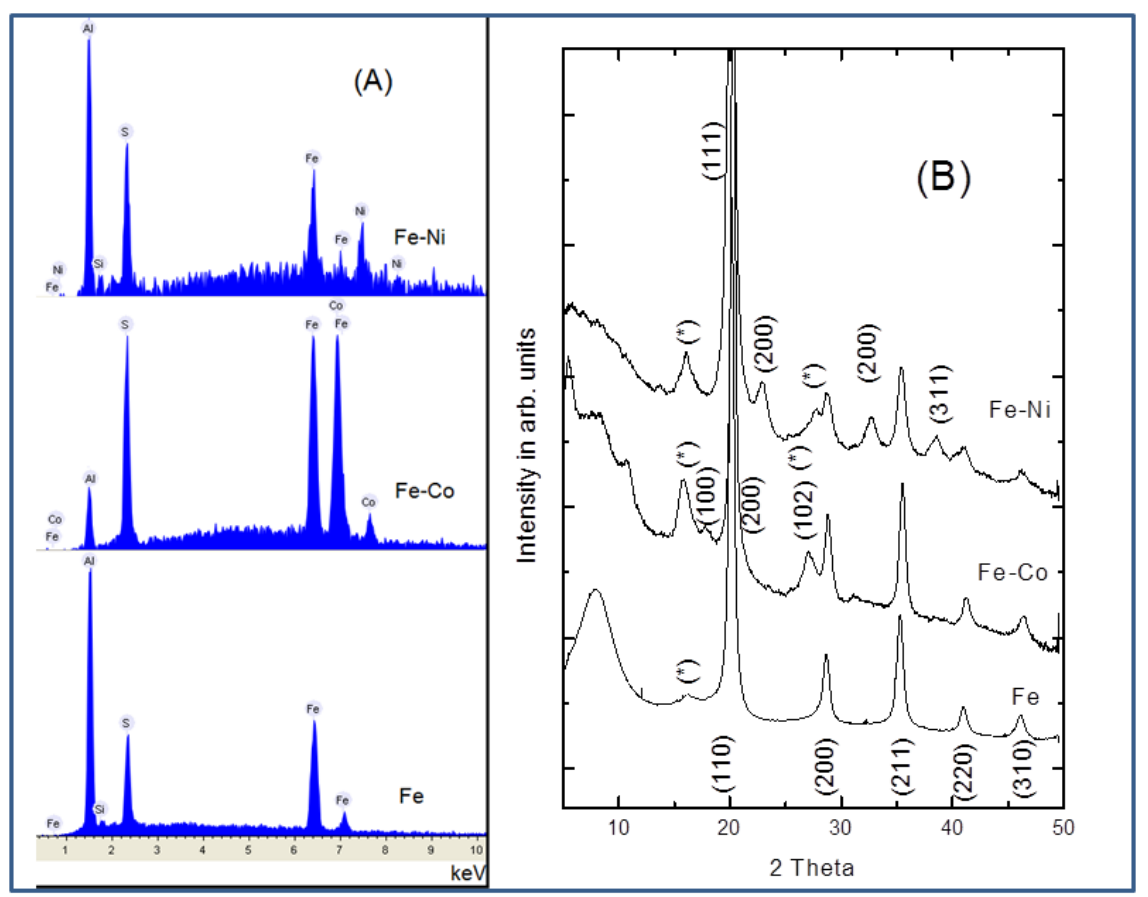

Fig. 2. (A) EDX analysis of deposited Fe, Fe-Co, and Fe-Ni wires 1:1; (B) XRD data for Fe, Fe-Co, and Fe-Ni wires. (*) denotes Fe oxide.

Table 1. Hyperfine parameters of the presented spectra

\begin{tabular}{lccccc}
\hline & \multicolumn{3}{c}{ Sextet } & \multicolumn{2}{c}{ Doublet } \\
\cline { 2 - 5 } \multicolumn{1}{c}{ Sample } & m.h.f. $\pm 0.5 \mathrm{~T}$ & $\begin{array}{c}\text { Isomer shift } \\
\pm 0.03 \mathrm{~mm} / \mathrm{s}\end{array}$ & $z \pm 0.1$ & $\begin{array}{c}\text { Isomer shift } \\
\pm 0.03 \mathrm{~mm} / \mathrm{s}\end{array}$ & $\begin{array}{c}\text { Quadrupole splitting } \\
\pm 0.03 \mathrm{~mm} / \mathrm{s}\end{array}$ \\
\hline Fe & 32.9 & 0.00 & 2.3 & 0.20 & 0.36 \\
Fe-Ni (1) & 33.2 & 0.00 & 1.9 & 0.22 & 0.31 \\
Fe-Ni (a) & 33.9 & 0.03 & 1.6 & 0.21 & 0.36 \\
Fe-Co (1) & 33.0 & 0.02 & 2.0 & 0.21 & 0.34 \\
Fe-Co (a) & 34.4 & 0.02 & 3.2 & 0.20 & 0.34 \\
\hline
\end{tabular}

The ${ }^{57} \mathrm{Fe}$ Mössbauer spectra obtained for each type of nanowires show the coexistence of doublet and sextet. Hyperfine parameters (magnetic hyperfine field m.h.f., isomer shift, and quadrupole splitting) of both subspectra are collected in Table 1. The presence of doublet can be explained either by: spontaneous surface oxidation process taking place at the interface between matrix and wire, occurring just after deposition. It can happen due to the fact that electrodeposition is performed in air and samples are also kept in oxidative conditions. The other option is that doublet comes from the grain boundary, which is less likely since the structure of nanowires is rather dense and smooth.

The ${ }^{57} \mathrm{Fe}$ hyperfine parameters (isomer shift and quadrupole splitting) obtained for the doublet, correspond well with the ones known for the corrosion product of metallic iron, mainly of $\mathrm{Fe}^{3+}$ oxidation state [19]. The hyperfine parameters of sextet m.h.f., isomer shift, and quadrupole splitting are very similar to these typical for $\alpha-F e$ [20]. It is in accordance with the relevant literature that the hyperfine parameters of Fe-Ni alloy are not too much different from the pure Fe ones, especially for the small concentration of $\mathrm{Ni}$ [21-25].

In case of $\mathrm{Fe}-\mathrm{Co}$ or $\mathrm{Fe}-\mathrm{Ni}$ wires, the ${ }^{57} \mathrm{Fe}$ magnetic hyperfine field is larger by about $1 \mathrm{~T}$ from the value corresponding to pure $\alpha$-Fe. This is in good agreement with the data obtained for Co-Fe multilayered system studied by CEMS [26, 27]. The presence of $\mathrm{Co}$ in the closest neighborhood of Fe causes the increase of magnetic hyperfine field of Fe due to strong modification of magnetic moment value from first coordination atoms.

The most important issue which we would like to discuss is the intensity ratio of the second and third lines in sextets that varies from 1.6 to 3.2 in respect of sample composition and intrinsic structure. The general relation between relative line intensities in ${ }^{57} \mathrm{Fe}$ sextet can be described as $3: z: 1: 1: z: 3$, where the $z$ value strongly depends on the relative orientation of the incident photon wave vector $\boldsymbol{k}$ with respect to the hyperfine magnetic field direction. For random oriented Fe magnetic moments, $z$ is equal to 2 , while for parallel or perpendicular it is 0 or 4 [28], respectively. The quantification of the $z$ value also allows to estimate the magnitude of the average square of the cosine: $\left\langle(\gamma \boldsymbol{m})^{2}\right\rangle=(4-z) /(4+z)$, where $\gamma$ stands for the unit vector which is parallel to the photon wave vector $\boldsymbol{k}$, and $\boldsymbol{m}$ means the unit vector parallel to the hyperfine external magnetic field vector. The brackets $<>$ denote averaging over the hyperfine field orientation. The value of $\left\langle(\gamma \boldsymbol{m})^{2}\right\rangle$ varies from 0 to 1 in respect of considered vectors relative 


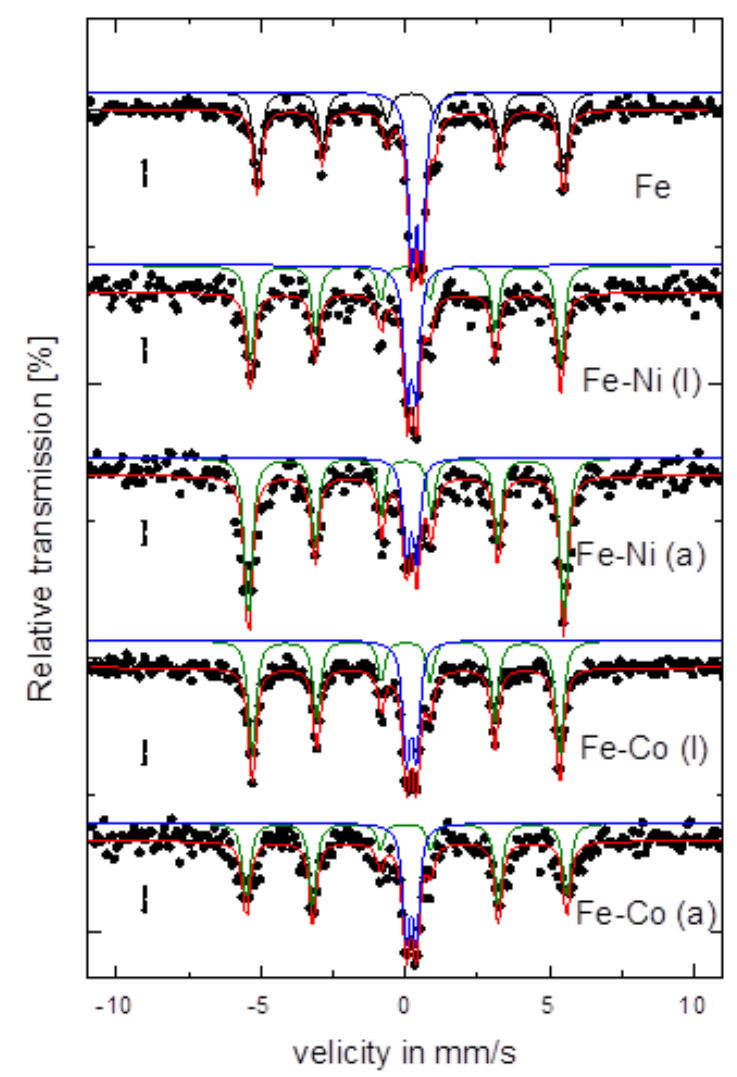

Fig. 3. ${ }^{57} \mathrm{Fe}$ Mössbauer spectra of $\mathrm{Fe}, \mathrm{Fe}-\mathrm{Ni}$, and $\mathrm{Co}-\mathrm{Fe}$ nanowires; (a) stand for alloy, (l) stand for layered.

orientations. For perfect perpendicular or parallel orientation of the magnetic moments to the matrix plane magnitude of $\left\langle(\gamma \boldsymbol{m})^{2}\right\rangle$ is 1 or 0 , respectively. Estimated values of the average square of the cosine for the presented samples vary from 0.11 to 0.43 and for some cases deviate significantly from the value expected for randomly distributed magnetic moments which is equal to 0.33 .

For ordered arrays of the nanowires of high aspect ratio, the domination of shape anisotropy can be expected and, therefore, nanowires should be predominately magnetized along the wire long axis. This was observed for the wires with a diameter smaller than $50 \mathrm{~nm}$ [29]. For larger wire diameters, the scenario can be different due to a high possibility of domain walls creations [30]. In this case the average value of $\left\langle(\gamma \boldsymbol{m})^{2}\right\rangle$ and thus relative line intensities will be modified and, as a result, different form values related to magnetization, oriented perpendicularly to the template plane. This situation is rather clear to understand for the wires with uniform composition. But the fabrication of elongated structures with distinguished layered inner texture, introduces additional contribution of shape anisotropy and for some system, extraordinary in plane or out-of-plane orientation of magnetic moments are expected especially at the interface regions. For the presented set of samples it is seen that Ni tends to orient $\mathrm{Fe}$ magnetic moments along the wire main axis, while Co rather opposite. Moreover, intermixing is important for the considered samples as it seems that more blended elements cause more specified magnetic moments arrangement in contrary to layered system. This observation needs more studies which are planned in the near future.

\section{Conclusion}

The analysis of the presented ${ }^{57} \mathrm{Fe}$ Mössbauer spectra shows that in respect of chemical composition (Co or Ni presence) and relative distribution of 3D elements in the wires (alloy or layered structures) observable average magnetic moments change their orientation. From the first results studied in the presented paper the cases are opposite to each other. Ni atoms affect Fe magnetization direction which is oriented mainly along the wires main easy axis. While Co presence in the structures organizes Fe magnetic moments perpendicular to the wire long axis.

Acknowledgment. The work was partially financed by the EU funds via the project with a contract number POPW.01.03.00-20-034/09-00 and by Polish National Grant UMO-2011/03/B/ST5/02691. The authors acknowledge Dr W. Olszewski and MSc E. Orzechowska, for laboratory assistance, and COMEF Scientific and Research Equipment company for SEM Microscope lending.

\section{References}

1. Ersen, O., Begin, S., Houlle, M., Amadou, J., Janowska, I., Greneche, J. M., Crucifix, C., \& Pham-Huu, C. (2008). Microstructural investigation of magnetic $\mathrm{CoFe}_{2} \mathrm{O}_{4}$ nanowires inside carbon nanotubes by electron tomography. Nano Lett., 8, 1033-1040. DOI: 10.1021/nl072714e.

2. Peter, L., Casik, A., Vad, K., Toth-Kadar, E., Pekker, A., \& Molnar, G. (2010). On the composition depth profile of electrodeposited Fe-Co-Ni alloys. Electrochim. Acta, 55, 4734-4741. DOI: 10.1016/j.electacta.2010.03.075.

3. Osaka, T. (2000). Electrodeposition of highly functional thin films for magnetic recording devices of the next century. Electrochim. Acta, 45, 3311-3321. DOI: 10.1016/S0013-4686(00)00407-2.

4. Quemper, J. M., Nicolas, S., Gilles, J. P., Grandchamp, J. P., Bosseboeuf, A., Bourouina, T., \& DufourGergam, E. (1999). Permalloy electroplating through photoresist molds. Sens. Actuator, 74, 1-4. DOI: 10.1016/S0924-4247 (98)00323-9.

5. Munoz, A. G. Schiefer, C., Nentwig, Th., Man, W. -Y., \& Kisker, E. (2007). Magneto impedance of electroplated $\mathrm{NiFeMo} / \mathrm{Cu}$ microwires for magnetic sensors. J. Phys. D-Appl. Phys., 40, 5013-5020. DOI: 10.1088/0022-3727/40/17/001.

6. Bauer, L. A., Birenbaum, N. S., \& Meyer, G. J. (2004). Biological applications of high aspect ratio nanoparticles. Mater. Chem., 14, 517-526. DOI: 10.1039/ b312655b.

7. Niemirowicz, K., Swiecicka, I., Wilczewska, A. Z., Misztalewska, I., Kalska-Szostko, B., Bienias, K., Bucki, R., \& Car, H. (2014). Gold-functionalized magnetic nanoparticles restrict growth of Pseudomonas aeruginosa. Int. J. Nanomed., 8(9), 2217-2224. DOI: $10.2147 / \mathrm{IJN}$.S56588.

8. Kalska-Szostko, B., Orzechowska, E., \& Wykowska, U. (2013). Organophosphorous modifications of 
multifunctional magnetic nanowires. Colloid Surf. B-Biointerfaces, 111, 509-516. DOI: 10.1016/j. colsurfb.2013.05.03.

9. Kalska-Szostko, B., \& Orzechowska, E. (2011). Preparation of magnetic nanowires modified with functional groups. Curr. Appl. Phys., 11(5), S103-S108. DOI: 10.1016/j.cap.2011.04.051.

10. Liu, X., Zangari, G., \& Shen, L. (2000). Electrodeposition of soft, high moment Co-Fe-Ni thin films. $J$. Appl. Phys., 87, 5410-5412. DOI: 10.1063/1.373359.

11. Kalska-Szostko, B., Brancewicz, E., Mazalski, P., Sveklo, J., Olszewski, W., Szymański, K., \& Sidor, A. (2009). Electrochemical deposition of nanowires in porous alumina. Acta Phys. Pol. A, 115, 542-544.

12. Kalska-Szostko, B., Brancewicz, E., Olszewski, W., Szymański, K., Sidor, A., Sveklo, J., \& Mazalski, P. (2009). Electrochemical preparation of magnetic nanowires. Solid State Phenom., 151, 190-196. DOI: 10.4028/www.scientific.net/SSP.151.190.

13. Kalska-Szostko, B., \& Orzechowska, E. (2011). Surface modification of core-shell nanowire with protein adsorption. Mater. Chem. Phys., 129, 256-260. DOI: 10.1016/j.matchemphys.2011.04.01.

14. Saedi, A., \& Ghorbani, M. (2005). Electrodeposition of Ni-Fe-Co alloy nanowire in modified AAO template. Mater. Chem. Phys., 91, 417-423. DOI: 10.1016/j. matchemphys.2004.12.001.

15. Kalska-Szostko, B., Wykowska, U., Piekut, K., \& Zambrzycka, E. (2013). Stability of iron (Fe) nanowires. Colloid Surf. A-Physiochem. Eng. Asp., 416, 66-72. DOI: 10.1016/j.colsurfa.2012.10.019.

16. Charlot, F., Gaffet, E., Zeghmati, B., Bernard, F., \& Niepce, J. C. (1999). Mechanically activated synthesis studied by X-ray diffraction in the Fe-Al system. Mater. Sci. Eng. A, 263, 279-288. DOI: 10.1016/ S0921-5093(98)01017-X

17. Matveev, V. V., Baranov, D. A., Yurkov, G. Y., Akatiev, N. G., Dotsenko, I. P., \& Gubin, S. P. (2006). Cobalt nanoparticles with preferential hcp structure: A confirmation by X-ray diffraction and NMR. Chem. Phys. Lett., 422, 402-405. DOI: 10.1016/j. cplett.2006.02.099.

18. Smirnov, A., Hausner, D., Laffers, R., Strongin, D. R., \& Schoonen, M. A. A. (2008). Abiotic ammonium formation in the presence of Ni-Fe metals and alloys and its implications for the Hadean nitrogen cycle. Geochem. Trans., 9(5), 1-20. DOI: 10.1186/14674866-9-5.

19. Greenwood, N. N., \& Gibb, T. C. (1971). Mössbauer spectroscopy. London: Chapman and Hall.

20. Korecki, J., \& Gradmann, U. (1985). In situ Mossbauer analysis of hyperfine interaction near Fe (110) surfaces and interfaces. Phys. Rev. Lett., 55(22), 2491-2494. DOI: 10.1103/PhysRevLett.55.2491.
21. Li, Q. F., Wang, J. B., Yan, Z. J., \& Xue, D. S. (2004). The effect of diameter on micro-magnetic properties of $\mathrm{Fe}_{0.68} \mathrm{Ni}_{0.32}$ nanowire arrays. J. Magn. Magn. Mater., 278, 323-327. DOI: 10.1016/j.jmmm.2003.12.1357.

22. de Oliveira, L. S., da Cunha, J. M. B., Spada, E. R., \& Hallouche, B. (2007). Mössbauer spectroscopy and magnetic properties in thin films of $\mathrm{Fe}_{x} \mathrm{Ni}_{100-x}$ electroplated on silicon (1 0 0). Appl. Surf. Sci., 254, 347-350. DOI: 10.1016/j.apsusc.2007.07.093.

23. Scorzelli, R. B., Souza Azevedo, I., Pereira, R. A., Perez, C. A. C., \& Fernandes, A. A. R. (1994). Mössbauer spectroscopy study of the metallic particles extracted from the Antarctic chondrite Allan Hills-769. In Proceedings NIPR Symposium Antarct. Meteorites 7, 31 May-2 June 1993 (pp. 299-303). Tokyo: National Institute of Polar Research.

24. Ping, J. Y., Rancourt, D. G., \& Dunlap, R. A. (1992) Physical basis and break down of hyperfine field distribution analysis in fcc Fe-Ni (5-70 at\%Fe). J. Magn. Magn. Mater., 103, 285-313. DOI: 10.1016/03048853(92)90201-X.

25. Guenzburger, D., \& Terrera, J. (2006). Theoretical investigation of Mössbauer hyperfine interactions in ordered $\mathrm{FeNi}$ and disordered Fe-Ni alloys. Hyperfine Interact., 168, 1159-1163. DOI: 10.1007/sI0751006-9416-0.

26. Häggström, L., Kalska, B., Blomquist, P., \& Wappling, R. (2002). Magnetic anisotropy and magnetic fields in bcc Fe/Co (001) superlattices. J. Alloy. Compd., 347, 252-258. DOI: 10.1016/S0925-8388(02)00762-4.

27. Kalska, B., Blomquist, P., Haggstrom, L., \& Wappling, R. (2001). Interface roughness/intermixing and magnetic moments in a $\mathrm{Fe} / \mathrm{Co}(001)$ superlattice. J. Phys.-Condens. Matter, 13, 2963-2970. DOI: 10.1088/0953-8984/13/13/310.

28. Kalska, B., Haggstrom, L., Blomquist, P., \& Wappling, R. (2000). Conversion electron Mössbauer spectroscopy studies of the magnetic moment distribution in $\mathrm{Fe} / \mathrm{V}$ multilayers. J. Phys.-Condens. Matter, 12, 539-548. DOI: 10.1088/0953-8984/12/5/302.

29. Hamrakulov, B., Kim, I., Lee, M. G., \& Park, B. H. (2009). Electrodeposited $\mathrm{Ni}, \mathrm{Fe}, \mathrm{Co}$ and $\mathrm{Cu}$ single and multilayer nanowires arrays on anodic aluminium oxide template. Trans. Nonferrous Met. Soc. China, 19, 83-87. DOI: 10.1016/S1003-6326(10)60250-6.

30. Leitao, D. C., Sousa, C. T., Ventura, J., Amaral, J. S., Carpineiro, F., Pirota, K. R., Vazquez, M., Sousa, J. B., \& Aroujo, J. P. (2008). Characterization of electrodeposited $\mathrm{Ni}$ and $\mathrm{Ni}_{80} \mathrm{Fe}_{20}$ nanowires. J. Non-Cryst. Solids, 354, 5241-5243. DOI: 10.1016/j.jnoncrysol.2008.05.088. 